\title{
The DESIGNER Approach Helps Decipher the Hypoglycemic Bioactive Principles of Artemisia dracunculus (Russian Tarragon)
}

Yongmei $\mathrm{Yu}^{\dagger}, \#$, Charlotte Simmler, ${ }^{\ddagger}$, Peter Kuhn ${ }^{\S}$, Alexander Poulev ${ }^{\S}$, Ilya Raskin ${ }^{\S}$, David Ribnicky ${ }^{\S}$, Z. Elizabeth Floyd ${ }^{\dagger}$, Guido F. Pauli

${ }^{\dagger}$ Pennington Biomedical Research Center, Louisiana State University System, Baton Rouge, LA, 70808, United States

Center for Natural Product Technologies, Program for Collaborative Research in the Pharmaceutical Sciences and Department of Pharmaceutical Sciences, University of Illinois at Chicago, 833 South Wood Street, Chicago, Illinois 60612, United States

${ }^{\S}$ Department of Plant Biology, Rutgers University, New Brunswick, New Jersey, 08901, United States

${ }^{\#}$ Equal Contributors

-SUPPORTING INFORMATION 


\section{TABLE OF CONTENTS}

\section{Supporting Information}

S1. NOMENCLATURE OF $A$. DRACUNCULUS EXTRACT AND BIOACTIVE CHALCONES. ...................................... 3

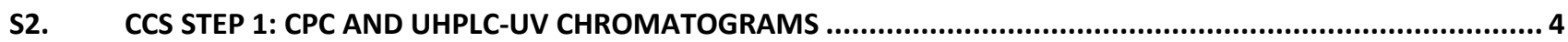

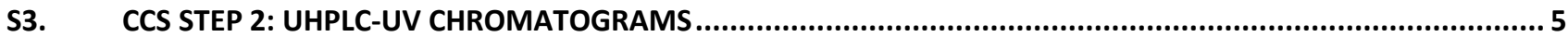

S4. EVALUATION OF SAMPLE RECOVERY FOR KOE AND THE RECONSTITUTED EXTRACT (RE)............................ 6

S5. COMPARATIVE UHPLC-UV CHROMATOGRAMS OF PMI-5011 CRUDE EXTRACT, KOE \& RE ........................... 6

S6. COMPARATIVE ${ }^{1}$ H NMR SPECTRA OF PMI-5011 CRUDE EXTRACT, KOE AND RE ....................................... 7

S7. UHPLC-UV QUANTITATIVE ANALYSIS OF (1) AND (2) IN THE DIFFERENT EXTRACTS.....................................8

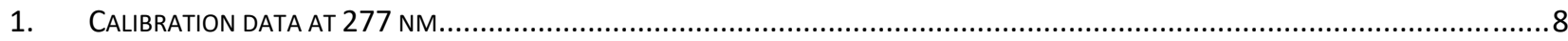

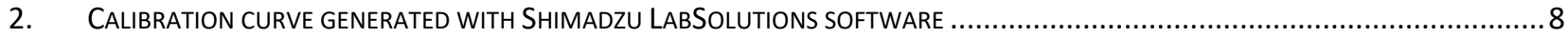

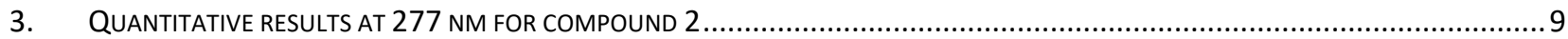

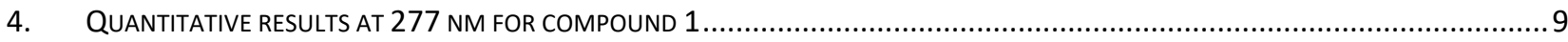

S8. UHPLC-UV QUANTITATIVE ANALYSIS OF ELEMICIN IN PMI-5011 EXTRACTS....................................... 10

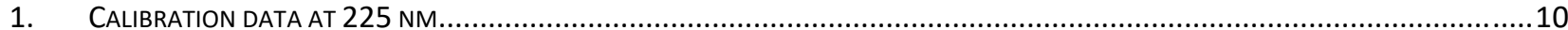

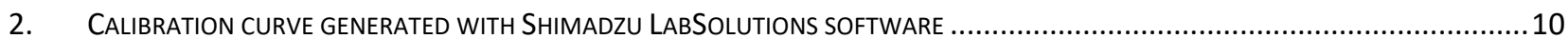

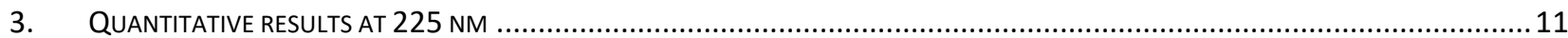

S9. ANNOTATED ${ }^{1} \mathrm{H}$ NMR OF 4-O-METHYLDAVIDIGENIN AND MS/MS SPECTRA OF BOTH ISOMERS ................. 12

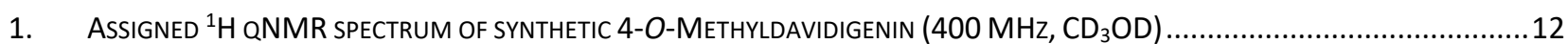

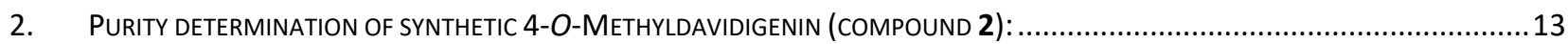

3. MS/MS SPECTRA OF 4-O-METHYLDAVIDIGENIN AND 4'-O-METHYLDAVIDIGENIN IN THE KOF (ESI+, 10-20 EV) .....................14

S10. COMPARATIVE ${ }^{1} \mathrm{H}$ NMR SPECTRA OF KOF WITH SYNTHETIC 4-O-METHYLDAVIDIGENIN............................ 15

S11. ANNOTATED ${ }^{1} \mathrm{H}$ NMR OF ELEMICIN AND MS/MS SPECTRA OF BOTH (ISO)-ELEMICIN ........................... 16

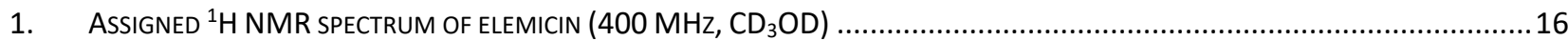

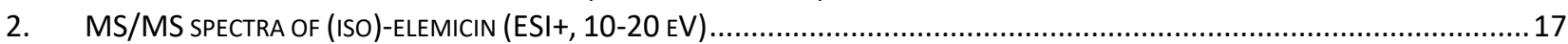

S12. UV SPECTRA OF METHYLDAVIDIGENIN ISOMERS, AND (ISO)-ELEMICIN.............................................. 18

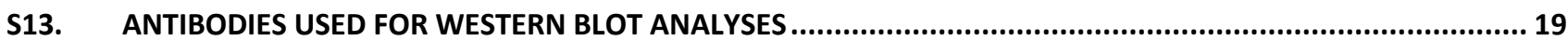

The NMR data collected for synthetic 4-O-methyldavidigenin (2), for the KOF containing the methyldavidigenin isomers (1) and (2), and elemicin (1D and 2D NMR data) as well as the different PMl-5011 extracts (CE, RE and KOE) are made freely available at https://doi.org/10.7910/DVN/QZH2DP (Harvard Dataverse). 


\section{S1. Nomenclature of $\boldsymbol{A}$. dracunculus extract and bioactive chalcones.}

The nomenclature of the plant extract, PMI-5011 originated from the original source of funding for our work with the Artemisia dracunculus, the company Phytomedics ("PMI") in New Jersey. In this scheme, "5011" refers to the laboratory of Drs. Ilya Raskin and David Ribnicky (laboratory/group "5", project "11").

The nomenclature of the Artemisia dracunculus chalcones was coined some 20 years ago in the laboratory of Drs. Ilya Raskin and David Ribnicky. The plant contained, among other chalcones, two isomers of 2,4-(D)ihydroxy-4-(M)ethoxy-dihydro(C)halcone, which were thus designated as DMC-1 (compound 1) and DMC-2 (compound 2).

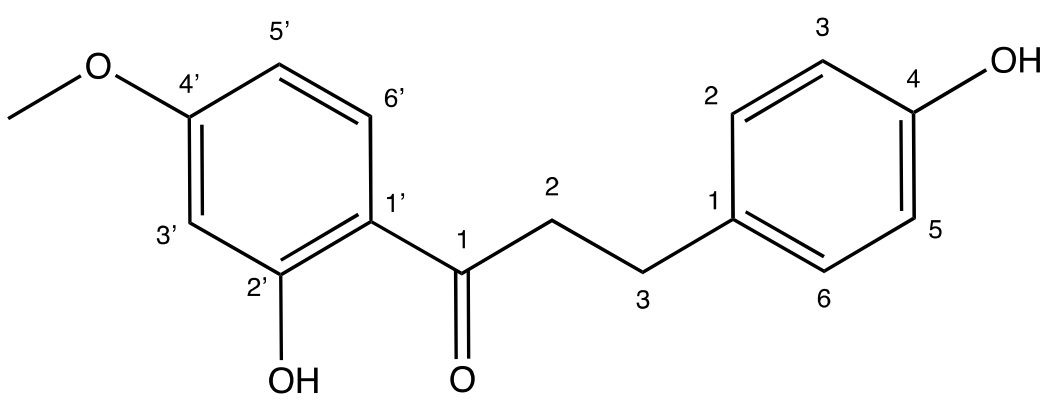

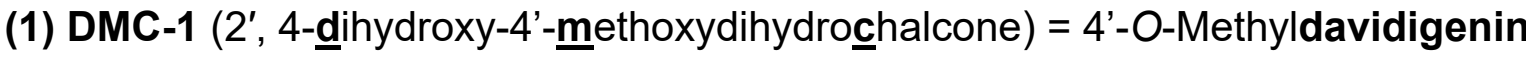

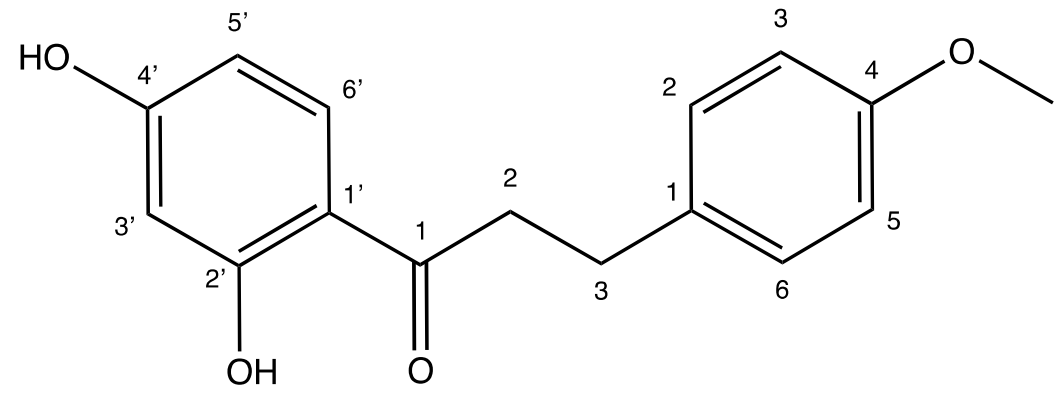

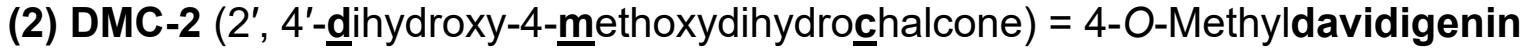<smiles>O=C(CCc1ccc(O)cc1)c1ccc(O)cc1O</smiles>

Davidigenin 


\section{S2. CCS step 1: CPC and UHPLC-UV Chromatograms}

A CCS Chromatogram of PMI-5011 CE

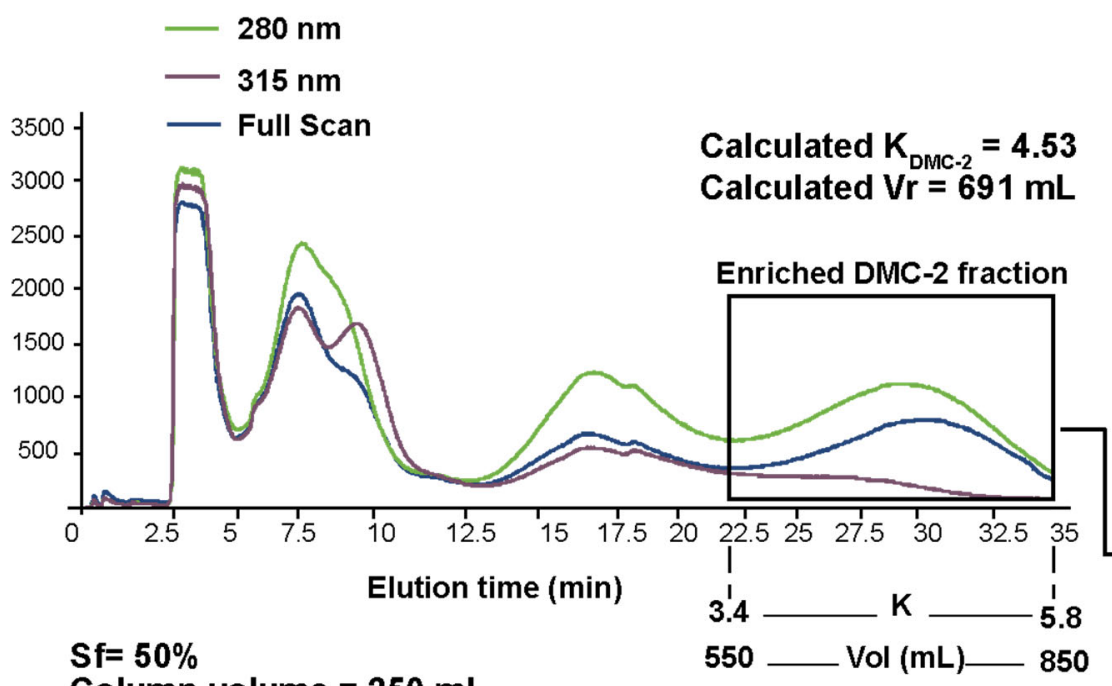

$\mathrm{Sf}=\mathbf{5 0 \%}$

Column volume $=250 \mathrm{~mL}$

Flow rate $=25 \mathrm{~mL} / \mathrm{min}$

Rotation speed $=\mathbf{2 8 0 0} \mathrm{rpm}$

Solvent System SS1, LP = mobile phase

Hexanes/Ethyl-Acetate/Ethanol/Water (5:4:4:4, v/v)
B UHPLC-UV Chromatograms (277nm) of fractions

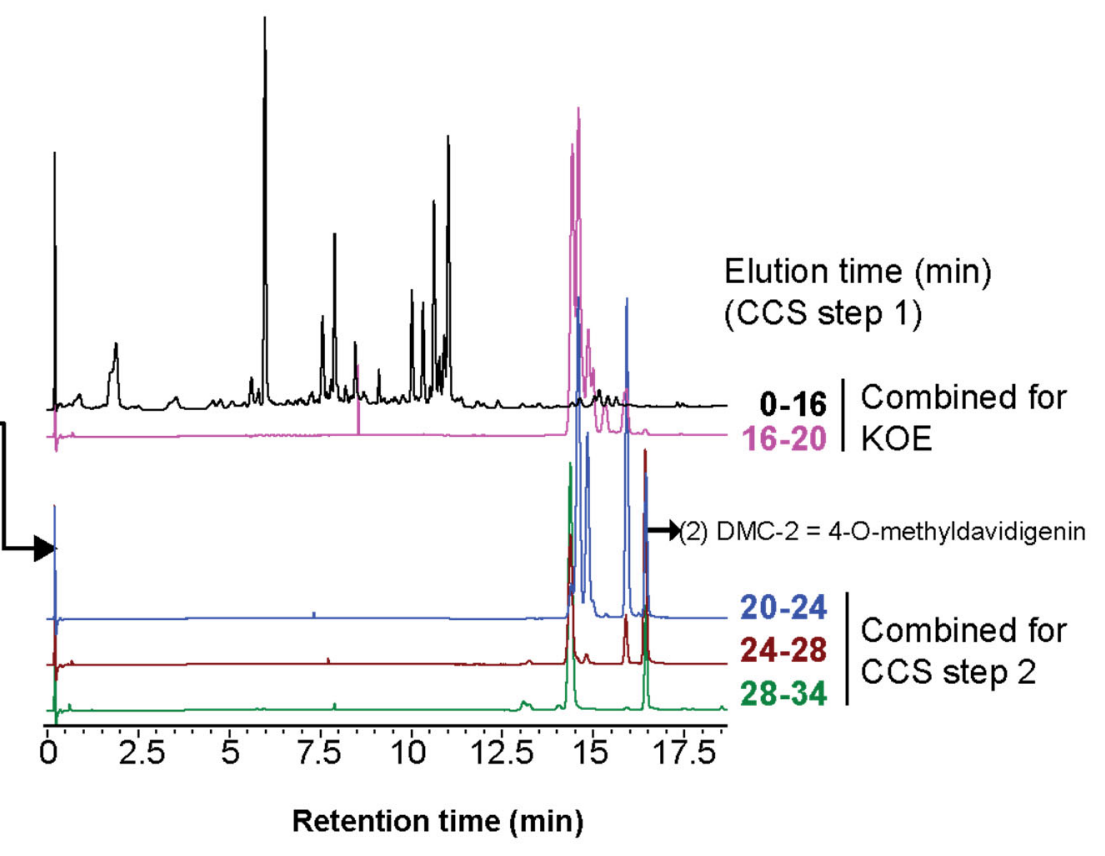




\section{S3. CCS step 2: UHPLC-UV chromatograms}

A Parameters of CCS step 2

B UHPLC-UV Chromatograms $(277 \mathrm{~nm})$ of fractions

\section{$\mathrm{Sf}=\mathbf{8 5} \%$}

Column volume $=\mathbf{3 0 0} \mathrm{mL}$

Flow rate $=1.5 \mathrm{~mL} / \mathrm{min}$

Rotation speed $=800 \mathrm{rpm}$

Solvent System SS2 =

Hexanes/Ethyl-Acetate/Methanol/Water (6:4:6:4 v/v)

LP = mobile phase

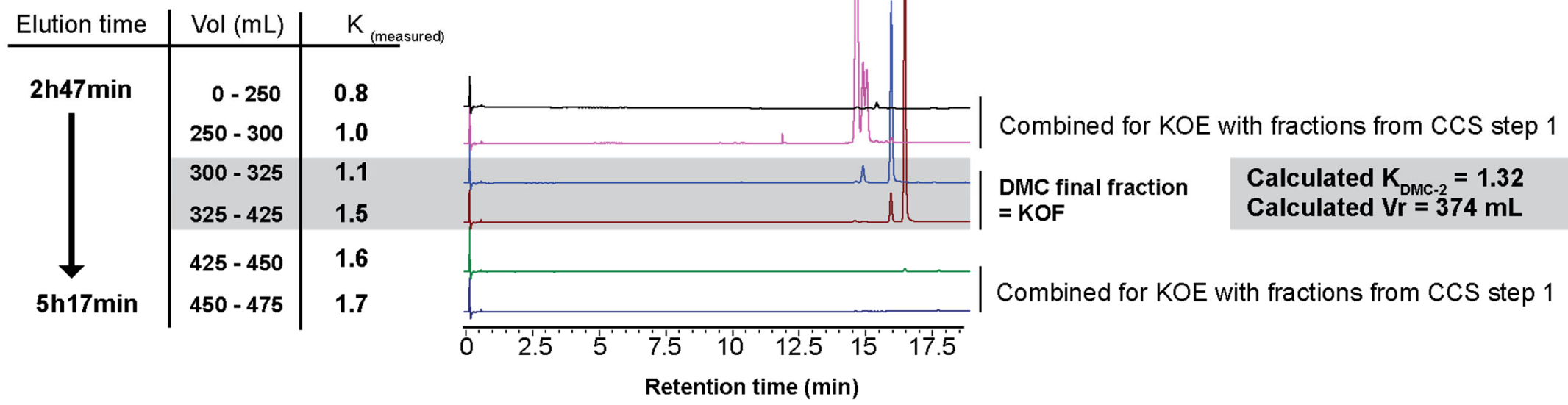


S4. Evaluation of sample recovery for KOE and the Reconstituted Extract (RE)

\begin{tabular}{|c|c|c|}
\hline & KOE & RE \\
\hline Quantity of PMI-5011 extract $(\mathbf{m g})$ & $\mathbf{1 3 0 6 . 9}$ & $\mathbf{1 2 7 4 . 4 6}$ \\
\hline CCS Step 1+2 quantity not injected $(\mathrm{mg})$ & 16.5 & 109.32 \\
\hline Total KOE (mg) & 1122.55 & \\
$\%$ w/w of recovered PMI 5011 & 97.8 & \multirow{2}{*}{ Not applicable } \\
Total KOF (mg) & 25.32 & \\
\hline Total w/w of recovered PMI 5011 & 2.20 & 1050.05 \\
$\%$ w/w of crude PMI5011 & 1147.87 & 87.83 \\
\hline
\end{tabular}

S5. Comparative UHPLC-UV chromatograms of PMI-5011 crude extract, KOE \& RE

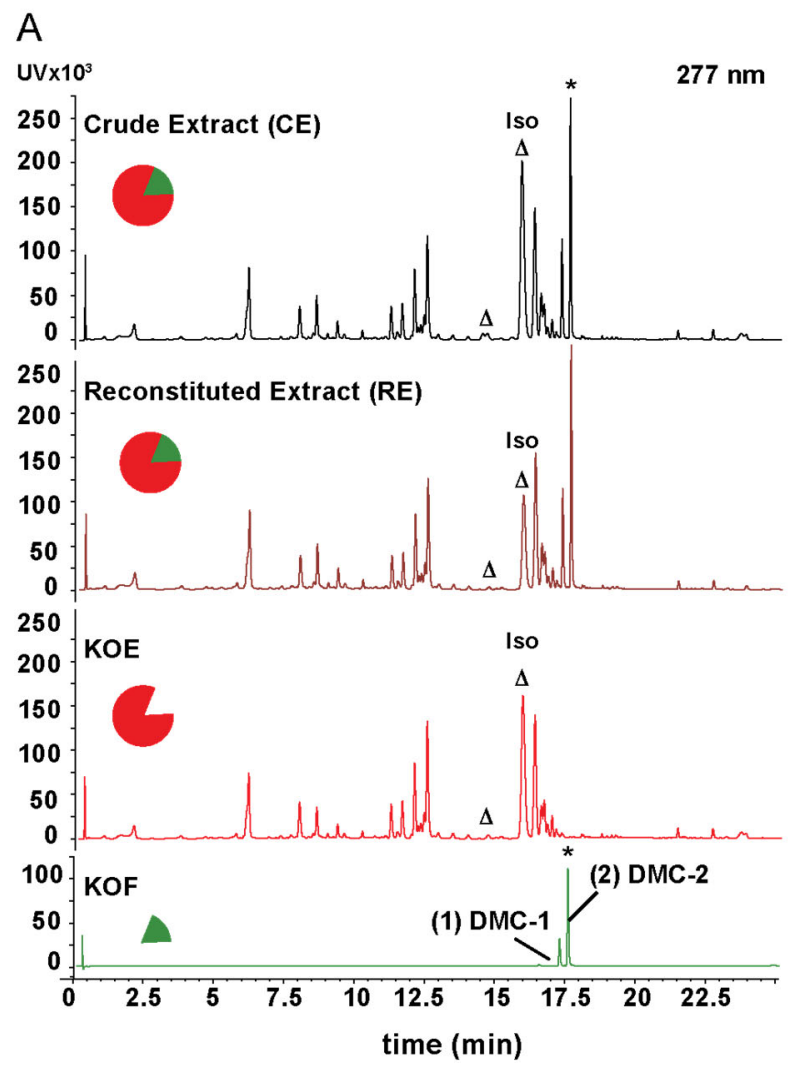

B
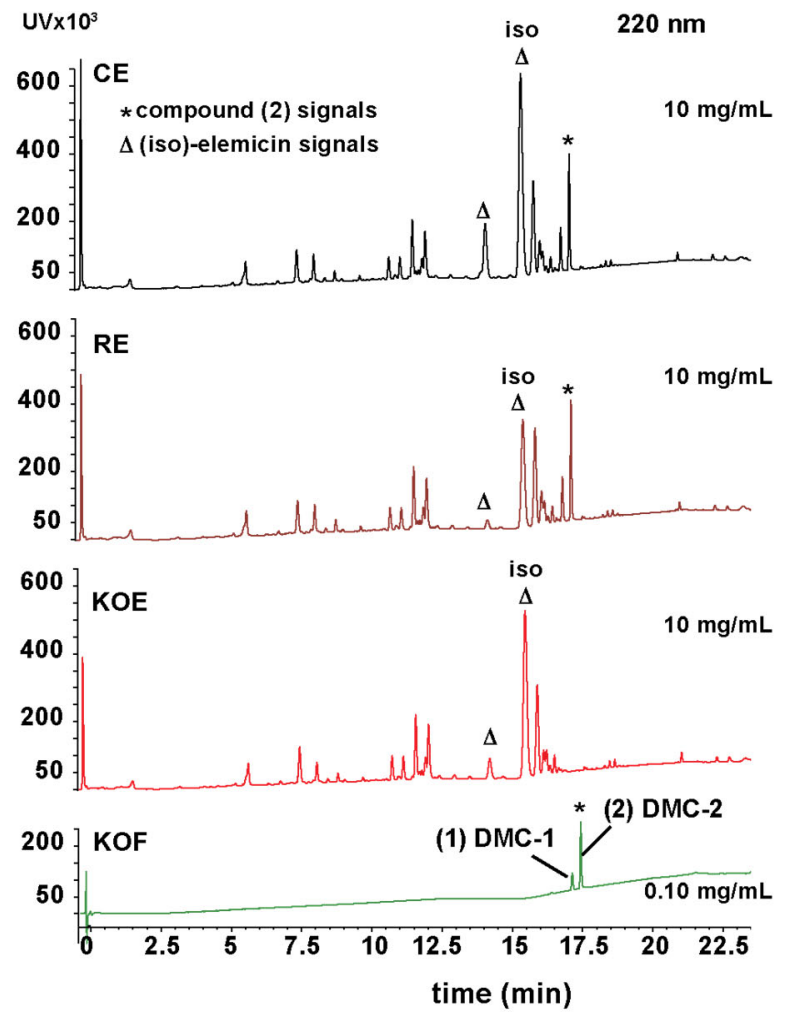

The depletion of elemicin in the reconstituted extracts (RE) and KOE is better detected at $220 \mathrm{~nm}$ (see also UV spectra of each compounds in S10). The RE is depleted in the volatile compounds (iso)-elemicin. 
S6. Comparative ${ }^{1} \mathrm{H}$ NMR spectra of PMI-5011 crude extract, KOE and RE
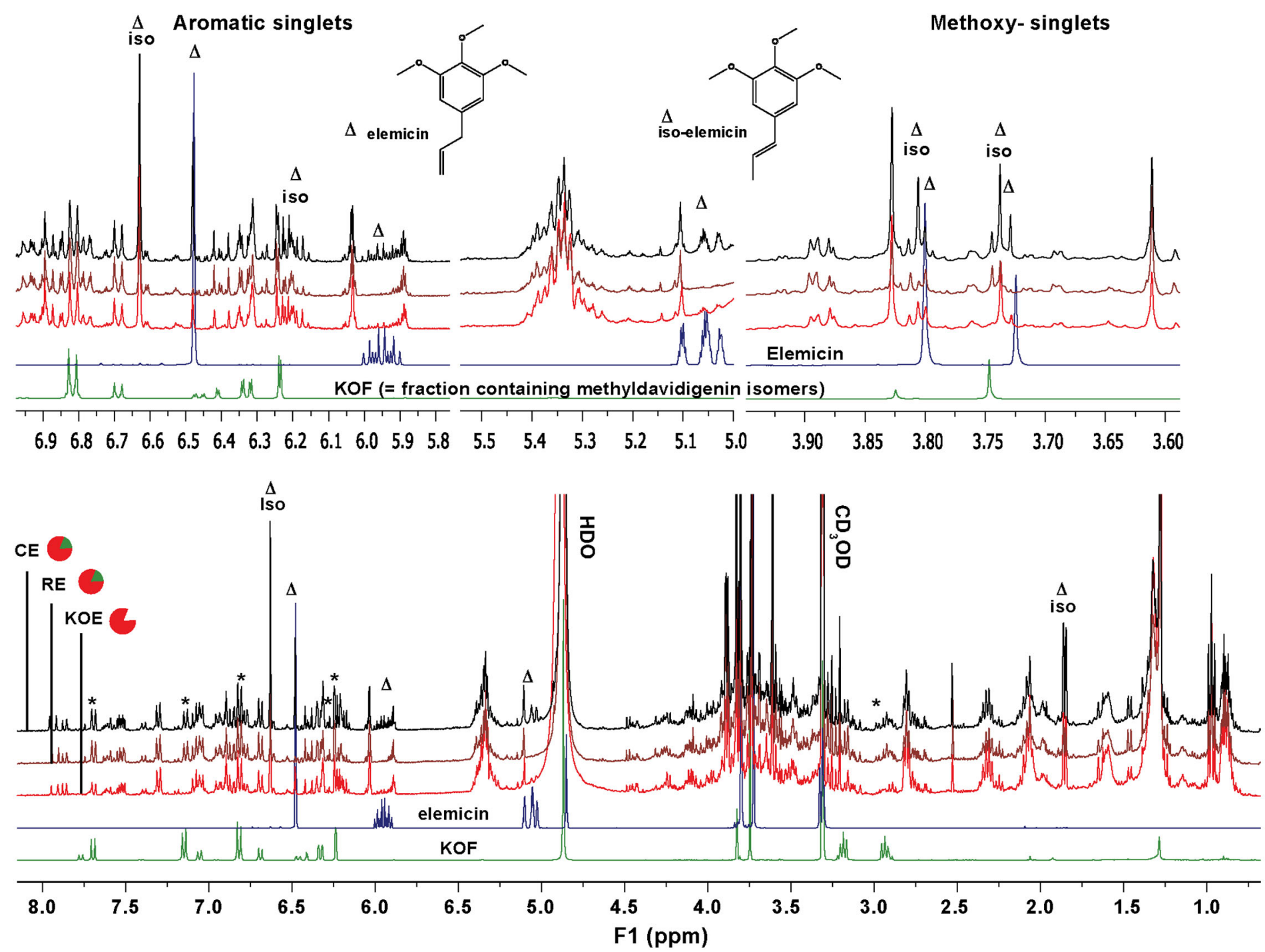

Data acquired under quantitative conditions at $400 \mathrm{MHz}$ in $\mathrm{CD}_{3} \mathrm{OD}$. $\mathrm{CE}=$ Crude Extract, $\mathrm{RE}=$ Reconstituted Extract, $\mathrm{KOE}=\mathrm{DMC}$ knock-out Extract. Also freely available at https://doi.org/10.7910/DVN/QZH2DP (Harvard Dataverse) 
S7. UHPLC-UV quantitative analysis of (1) and (2) in the different extracts

1. Calibration data at $277 \mathrm{~nm}$

\begin{tabular}{|l|l|l|l|l|l|l|l|}
\hline Sample Name & $\begin{array}{c}\text { Ret. } \\
\text { Time }\end{array}$ & \multicolumn{1}{|c|}{ Area } & $\begin{array}{c}\text { Conc. } \\
\text { (ug/mL) }\end{array}$ & Std. Conc. & \multicolumn{1}{|c|}{ Area\% } & $\begin{array}{c}\text { Accuracy } \\
\text { [\%] }\end{array}$ & \multicolumn{1}{c|}{ S/N } \\
\hline DMC2_1562_3 & 17.491 & 68,710 & 15.30 & 15.6 & 99.066 & 98.1 & 24.06 \\
\hline DMC2_1562_2 & 17.495 & 67,542 & 15.05 & 15.6 & 95.93 & 96.5 & 21.64 \\
\hline DMC2_1562_1 & 17.491 & 68,387 & 15.23 & 15.6 & 95.808 & 97.6 & 18.83 \\
\hline DMC2_3062_1 & 17.499 & 142,963 & 31.03 & 31.25 & 98.120 & 99.3 & 44.42 \\
\hline DMC2_3062_3 & 17.494 & 144,540 & 31.37 & 31.25 & 98.801 & 100.4 & 47.40 \\
\hline DMC2_3062_2 & 17.497 & 141,970 & 30.82 & 31.25 & 99.873 & 98.6 & 49.37 \\
\hline DMC2_6025_3 & 17.502 & 282,838 & 60.66 & 62.5 & 99.238 & 97.1 & 124.36 \\
\hline DMC2_6025_1 & 17.499 & 281,152 & 60.31 & 62.5 & 98.995 & 96.5 & 93.75 \\
\hline DMC2_6025_2 & 17.504 & $\mathbf{2 8 3 , 9 4 3}$ & 60.90 & 62.5 & 99.291 & 97.4 & 129.43 \\
\hline DMC2_12500_2 & 17.496 & 587,147 & 125.13 & 125 & 99.609 & 100.1 & 213.33 \\
\hline DMC2_12500_1 & 17.495 & 588,909 & 125.5 & 125 & 99.375 & 100.4 & 190.20 \\
\hline DMC2_12500_3 & 17.494 & 590,286 & 125.79 & 125 & 99.602 & 100.6 & 223.90 \\
\hline DMC2_25000_2 & 17.486 & $1,198,163$ & 254.57 & 250 & 99.648 & 101.8 & 378.77 \\
\hline DMC2_25000_3 & 17.484 & $1,193,199$ & 253.52 & 250 & 99.852 & 101.4 & 411.47 \\
\hline DMC2_25000_1 & 17.484 & $1,190,662$ & 252.98 & 250 & 99.826 & 101.2 & 479.27 \\
\hline DMC2_50000_2 & 17.458 & $2,355,102$ & 499.66 & 500 & 99.905 & 99.9 & 804.67 \\
\hline DMC2_50000_3 & 17.461 & $\mathbf{2 , 3 5 2 , 3 8 8}$ & 499.09 & 500 & 99.974 & 99.8 & 851.06 \\
\hline DMC2_50000_1 & 17.464 & $\mathbf{2 , 3 3 8 , 4 1 9}$ & 496.13 & 500 & 99.827 & 99.2 & 746.33 \\
\hline Average & $\mathbf{1 7 . 4 8 9}$ & $\mathbf{7 7 0 , 9 0 7}$ & $\mathbf{1 6 4 . 0 6}$ & & $\mathbf{9 9 . 0 4 1}$ & $\mathbf{9 9 . 2}$ & $\mathbf{2 6 9 . 5 7}$ \\
\hline Maximum & $\mathbf{1 7 . 5 0 4}$ & $\mathbf{2 , 3 5 5 , 1 0 2}$ & $\mathbf{4 9 9 . 6 6}$ & & $\mathbf{9 9 . 9 7 4}$ & $\mathbf{1 0 1 . 8}$ & $\mathbf{8 5 1 . 0 6}$ \\
\hline Minimum & $\mathbf{1 7 . 4 5 8}$ & $\mathbf{6 7 , 5 4 2}$ & $\mathbf{1 5 . 0 5}$ & & $\mathbf{9 5 . 8 0 8}$ & $\mathbf{9 6 . 5}$ & $\mathbf{1 8 . 8 3}$ \\
\hline Std. Dev. & $\mathbf{0 . 0 1 3}$ & $\mathbf{8 2 1 , 8 7 6}$ & $\mathbf{1 7 4 . 1 1 1}$ & & $\mathbf{1 . 2 4 7}$ & $\mathbf{1 . 6 9 2}$ & $\mathbf{2 8 1 . 1 8 7}$ \\
\hline
\end{tabular}

Calculated Limit of Detection $(\mathrm{LOD})=2.06 \pm 0.26 \mu \mathrm{g} / \mathrm{mL}$

Calculated Limit of Quantitation (LOQ) $=6.23 \pm 0.80 \mu \mathrm{g} / \mathrm{mL}$

2. Calibration curve generated with Shimadzu LabSolutions software

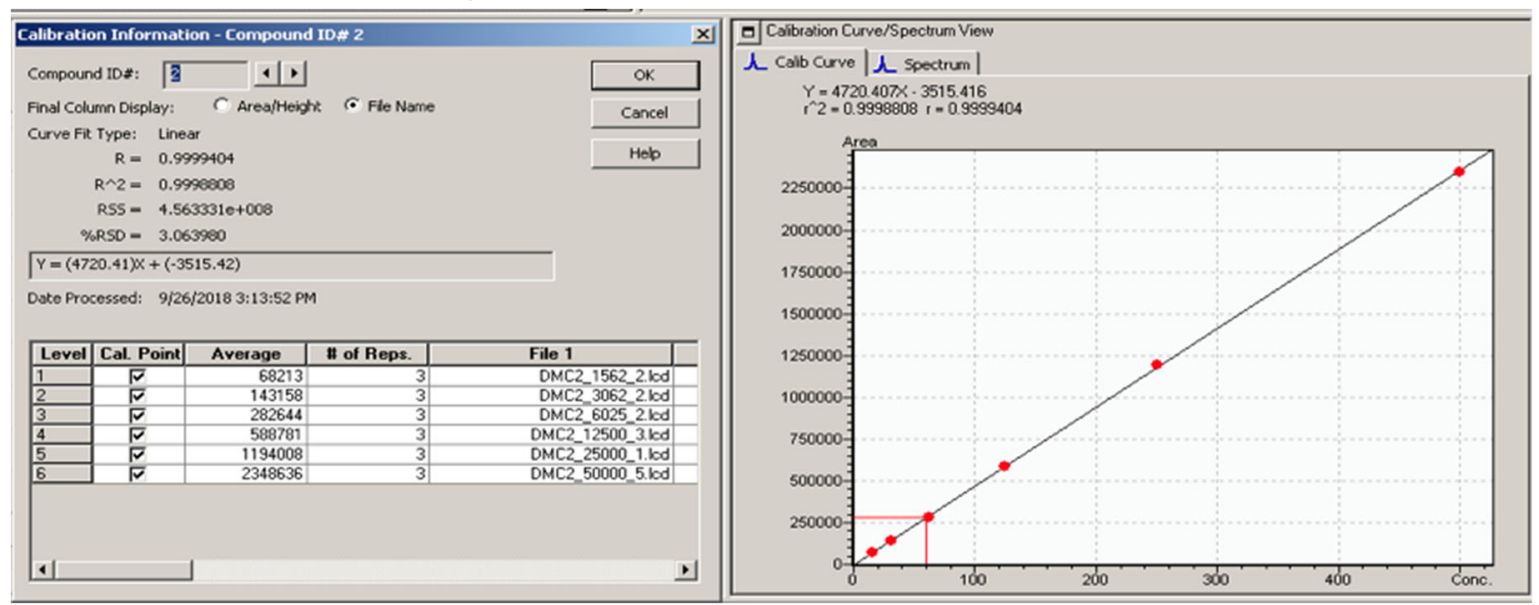


3. Quantitative results at $277 \mathrm{~nm}$ for (2) 4-O-methyldavidigenin

\begin{tabular}{|c|c|c|c|c|c|c|}
\hline Sample Name & Ret. Time & Area & Conc. (ug/mL) & \multicolumn{3}{|c|}{$\% \mathrm{w} / \mathrm{w}$ of analyzed sample } \\
\hline & & & & & Mean & Stdev \\
\hline PMI 5011 RE_1 & 17.487 & 837928 & 178.26 & 1.78 & \multirow{3}{*}{1.77} & \multirow{3}{*}{0.01} \\
\hline PMI 5011 RE_2 & 17.496 & 835535 & 177.75 & 1.78 & & \\
\hline PMI 5011 RE_3 & 17.488 & 828711 & 176.30 & 1.76 & & \\
\hline PMI 5011 CE_1 & 17.486 & 824874 & 175.49 & 1.75 & \multirow{2}{*}{1.76} & \multirow{2}{*}{0.00} \\
\hline PMI 5011 CE_2 & 17.484 & 828182 & 176.19 & 1.76 & & \\
\hline PM 5011 KOE_1 & 17.505 & $-1,069$ & $\angle L O D$ & $<L O D$ & \multirow{3}{*}{$\begin{array}{c}\text { Not } \\
\text { applicable }\end{array}$} & \multirow{3}{*}{$\begin{array}{c}\text { Not } \\
\text { applicable }\end{array}$} \\
\hline PMI 5011_KOE_2 & 17.493 & 64 & $<L O D$ & $<\mathrm{LOD}$ & & \\
\hline PMI 5011_KOE_3 & 17.506 & 314 & $\angle L O D$ & $<L O D$ & & \\
\hline KOF_1 & 17.500 & 344050 & 73.63 & 68.18 & \multirow{3}{*}{68.73} & \multirow{3}{*}{0.49} \\
\hline KOF_2 & 17.505 & 347880 & 74.44 & 68.93 & & \\
\hline KOF_3 & 17.498 & 348741 & 74.62 & 69.09 & & \\
\hline
\end{tabular}

All extracts were analyzed at $10 \mathrm{mg} / \mathrm{mL}$, the fraction (KOF) containing compounds (1) and (2) was analyzed at 0.108 $\mathrm{mg} / \mathrm{mL}$. The reconstituted extract (RE), contains the same amount of compound (2) than the original PMI-5011 crude extract (CE). Compound 2 is not detectable in the KOE.

\section{Quantitative results at $277 \mathrm{~nm}$ for (1) 4'-O-methyldavidigenin}

\begin{tabular}{|c|c|c|c|c|c|c|}
\hline Sample Name & Ret. Time & Area & $\begin{array}{c}\text { Conc. } \\
\text { (ug/mL) }\end{array}$ & \multicolumn{3}{|c|}{$\% \mathrm{w} / \mathrm{w}$ of analyzed sample } \\
\hline & & & & & Mean & Stdev \\
\hline PMI 5011 RE_1 & 17.19 & 380,056 & 81.26 & 0.813 & \multirow{3}{*}{0.81} & \multirow{3}{*}{0.00} \\
\hline PMI 5011 RE_2 & 17.20 & 378,701 & 80.97 & 0.810 & & \\
\hline PMI 5011 RE_3 & 17.19 & 381,310 & 81.52 & 0.815 & & \\
\hline PMI 5011 CE_1 & 17.18 & 376,300 & 80.46 & 0.805 & \multirow{2}{*}{0.81} & \multirow{2}{*}{0.00} \\
\hline PMI 5011 CE_2 & 17.19 & 378,388 & 80.90 & 0.809 & & \\
\hline PM 5011 KOE_1 & 17.20 & 17,467 & $4.45<\mathrm{LOQ}$ & 0.044 & \multirow{3}{*}{0.04} & \multirow{3}{*}{0.01} \\
\hline PMI 5011_KOE_2 & 17.19 & 17,841 & $4.52<\mathrm{LOQ}$ & 0.045 & & \\
\hline PMI 5011_KOE_3 & 17.21 & 9,848 & $2.83<\mathrm{LOQ}$ & 0.028 & & \\
\hline KOF_1 & 17.21 & 108,261 & 23.68 & 21.93 & \multirow{3}{*}{21.86} & \multirow{3}{*}{0.09} \\
\hline KOF_2 & 17.20 & 107,418 & 23.50 & 21.76 & & \\
\hline KOF_3 & 17.20 & 108,083 & 23.64 & 21.89 & & \\
\hline
\end{tabular}

All extracts were analyzed at $10 \mathrm{mg} / \mathrm{mL}$, the KOF (containing both compounds (1) and (2)) was analyzed at 0.108 $\mathrm{mg} / \mathrm{mL}$. The reconstituted extract (RE) contains the same amount of compound 1 than the original PMI-5011 crude extract (CE). The concentration of compound 1 was found to be below the limit of quantification in the KOE.

Considering that both compounds $\mathbf{1}$ and $\mathbf{2}$ are isomers, the calibration curve obtained for compound $\mathbf{2}$ was used to determine the concentration of compound 1. 
S8. UHPLC-UV quantitative analysis of elemicin in PMI-5011 extracts

1. Calibration data at $225 \mathrm{~nm}$

\begin{tabular}{|l|r|r|r|r|r|r|r|}
\hline \multicolumn{1}{|c|}{ Sample Name } & Rt & \multicolumn{1}{c|}{ Area } & \multicolumn{1}{c|}{$\begin{array}{c}\text { Conc. } \\
\text { (ug/mL) }\end{array}$} & \multicolumn{1}{c|}{$\begin{array}{c}\text { Std. } \\
\text { Conc. }\end{array}$} & \multicolumn{1}{c|}{$\begin{array}{c}\text { Area } \\
\text { \% }\end{array}$} & \multicolumn{1}{c|}{$\begin{array}{c}\text { Accuracy } \\
\text { [\%] }\end{array}$} & \multicolumn{1}{c|}{ S/N } \\
\hline Elemicin_0.0078_3 & 14.39 & 25,489 & 7.51 & 7.80 & 100.00 & 96.20 & 1.22 \\
\hline Elemicin_0.0078_1 & 14.39 & 25,747 & 7.58 & 7.80 & 100.00 & 97.20 & 1.20 \\
\hline Elemicin_0.0078_2 & 14.39 & 25,492 & 7.51 & 7.80 & 100.00 & 96.20 & 1.26 \\
\hline Elemicin_0.0156_1 & 14.40 & 55,774 & 16.10 & 15.60 & 100.00 & 103.20 & 2.56 \\
\hline Elemicin_0.0156_2 & 14.38 & 55,090 & 15.90 & 15.60 & 100.00 & 102.00 & 2.87 \\
\hline Elemicin_0.0156_3 & 14.38 & 54,675 & 15.79 & 15.60 & 100.00 & 101.20 & 2.65 \\
\hline Elemicin_0.0312_1 & 14.38 & 109,408 & 31.31 & 31.20 & 100.00 & 100.40 & 4.64 \\
\hline Elemicin_0.0312_2 & 14.38 & 109,324 & 31.29 & 31.20 & 100.00 & 100.30 & 4.54 \\
\hline Elemicin_0.0312_3 & 14.38 & 109,406 & 31.31 & 31.20 & 100.00 & 100.40 & 5.35 \\
\hline Elemicin_0.0625_1 & 14.39 & 219,436 & 62.53 & 62.50 & 100.00 & 100.00 & 9.84 \\
\hline Elemicin_0.0625_2 & 14.39 & 219,979 & 62.68 & 62.50 & 100.00 & 100.30 & 9.62 \\
\hline Elemicin_0.0625_3 & 14.38 & 217,518 & 61.99 & 62.50 & 100.00 & 99.20 & 8.57 \\
\hline Elemicin_0.125_1 & 14.38 & 434,242 & 123.47 & 125.00 & 100.00 & 98.80 & 18.68 \\
\hline Elemicin_0.125_2 & 14.38 & 433,409 & 123.24 & 125.00 & 100.00 & 98.60 & 16.81 \\
\hline Elemicin_0.125_3 & 14.29 & 449,836 & 127.90 & 125.00 & 100.00 & 102.30 & 15.17 \\
\hline Elemicin_0.250_1 & 14.28 & 879,616 & 249.83 & 250.00 & 100.00 & 99.90 & 32.93 \\
\hline Elemicin_0.250_2 & 14.27 & 882,063 & 250.52 & 250.00 & 100.00 & 100.20 & 34.74 \\
\hline Elemicin_0.250_3 & 14.28 & 879,709 & 249.85 & 250.00 & 100.00 & 99.90 & 29.17 \\
\hline Average & $\mathbf{1 4 . 3 6}$ & $\mathbf{2 8 8 , 1 2 3}$ & $\mathbf{8 2 . 0 2}$ & & $\mathbf{1 0 0 . 0 0}$ & $\mathbf{9 9 . 8 0}$ & $\mathbf{1 1 . 2 1}$ \\
\hline Maximum & $\mathbf{1 4 . 4 0}$ & $\mathbf{8 8 2 0 6 3}$ & $\mathbf{2 5 0 . 5 2}$ & & $\mathbf{1 0 0 . 0 0}$ & $\mathbf{1 0 3 . 2 0}$ & $\mathbf{3 4 . 7 4}$ \\
\hline Minimum & $\mathbf{1 4 . 2 7}$ & $\mathbf{2 5 4 8 9}$ & $\mathbf{7 . 5 1}$ & & $\mathbf{1 0 0 . 0 0}$ & $\mathbf{9 6 . 2 0}$ & $\mathbf{1 . 2 0}$ \\
\hline Std. Dev. & $\mathbf{0 . 0 4}$ & $\mathbf{3 0 6 8 8 0 . 8 0}$ & $\mathbf{8 7 . 0 6}$ & & $\mathbf{0 . 0 0}$ & $\mathbf{1 . 8 9}$ & $\mathbf{1 1 . 1 2}$ \\
\hline
\end{tabular}

2. Calibration curve generated with Shimadzu LabSolutions software

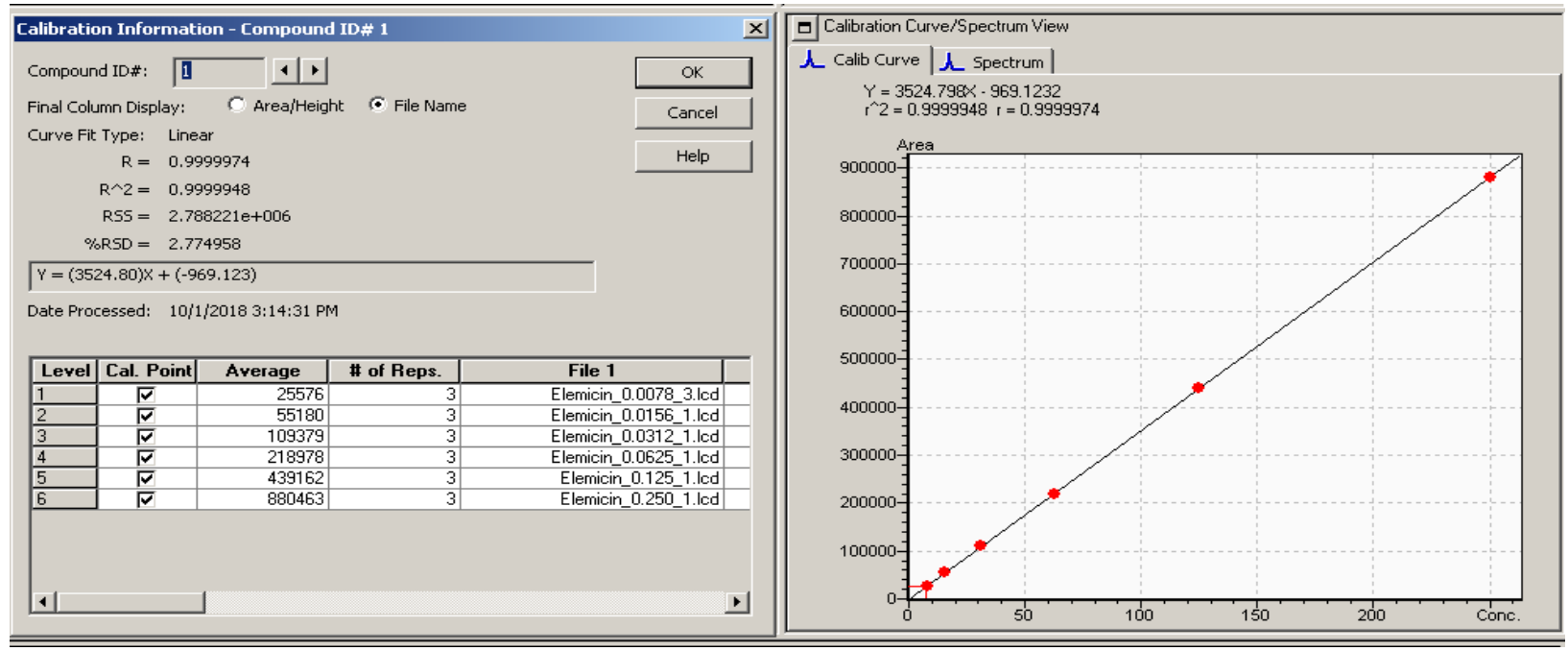


3. Quantitative results at $225 \mathrm{~nm}$

\begin{tabular}{|c|c|c|c|c|c|c|}
\hline Sample Name & Ret. Time & Area & Conc. (ug/mL) & \multicolumn{3}{|c|}{$\%$ w/w of analyzed sample } \\
\hline & & & & & Mean & Stdev \\
\hline PMI 5011 RE_1 & 14.55 & 139,514 & 39.86 & 0.40 & \multirow{3}{*}{$0.40<\mathrm{LOQ}$} & \multirow{3}{*}{0.00} \\
\hline PMI 5011 RE_2 & 14.56 & 138,592 & 39.59 & 0.40 & & \\
\hline PMI 5011 RE_3 & 14.54 & 138,719 & 39.63 & 0.40 & & \\
\hline PMI 5011 CE_1 & 14.51 & 767,101 & 217.91 & 2.18 & \multirow{2}{*}{2.16} & \multirow{2}{*}{0.03} \\
\hline PMI 5011 CE_2 & 14.51 & 750,568 & 213.21 & 2.13 & & \\
\hline PM 5011 KOE_1 & 14.57 & 325,323 & 92.57 & 0.93 & \multirow{3}{*}{0.93} & \multirow{3}{*}{0.00} \\
\hline PMI 5011_KOE_2 & 14.54 & 326,512 & 92.91 & 0.93 & & \\
\hline PMI 5011_KOE_3 & 14.58 & 326,507 & 92.91 & 0.93 & & \\
\hline
\end{tabular}

Elemicin is 2.3 times less concentrated in $\mathrm{KOE}$ and at least 5.4 times less concentrated in the reconstituted extract (RE) compared to the original PMI 5011 crude extract.

Calculated Limit of Detection $($ LOD $)=22.29 \pm 2.78 \mu \mathrm{g} / \mathrm{mL}$

Calculated Limit of Quantitation (LOQ) $=67.55 \pm 8.45 \mu \mathrm{g} / \mathrm{mLI}$ 


\section{S9. Annotated ${ }^{1} \mathrm{H}$ NMR of 4-O-methyldavidigenin and MS/MS spectra of both isomers}

1. Assigned ${ }^{1} \mathrm{H}$ qNMR spectrum of synthetic 4-O-methyldavidigenin syn, DMC-2 (400 MHz, CD $\left.30 D\right)$

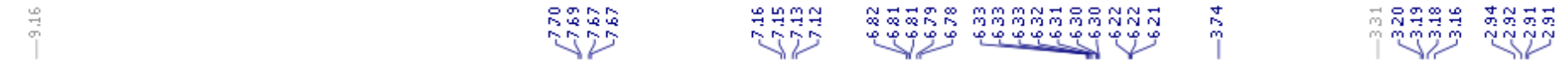

$\mathbb{C}=2.47 \mathrm{mg}$ in $275 \mathrm{uL}, 200 \mathrm{uL}$ used to dilute $2.02 \mathrm{mg}$ of DMC-2

DMC2 ${ }^{1} \mathrm{HNMR}$ spectrum (400MHz, CD3OD)
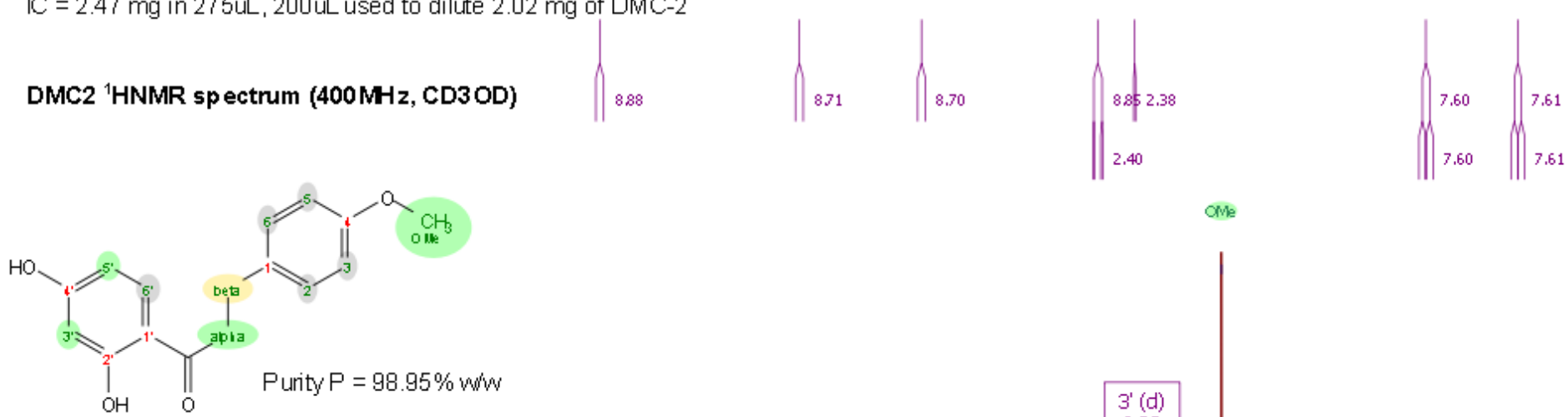

ome

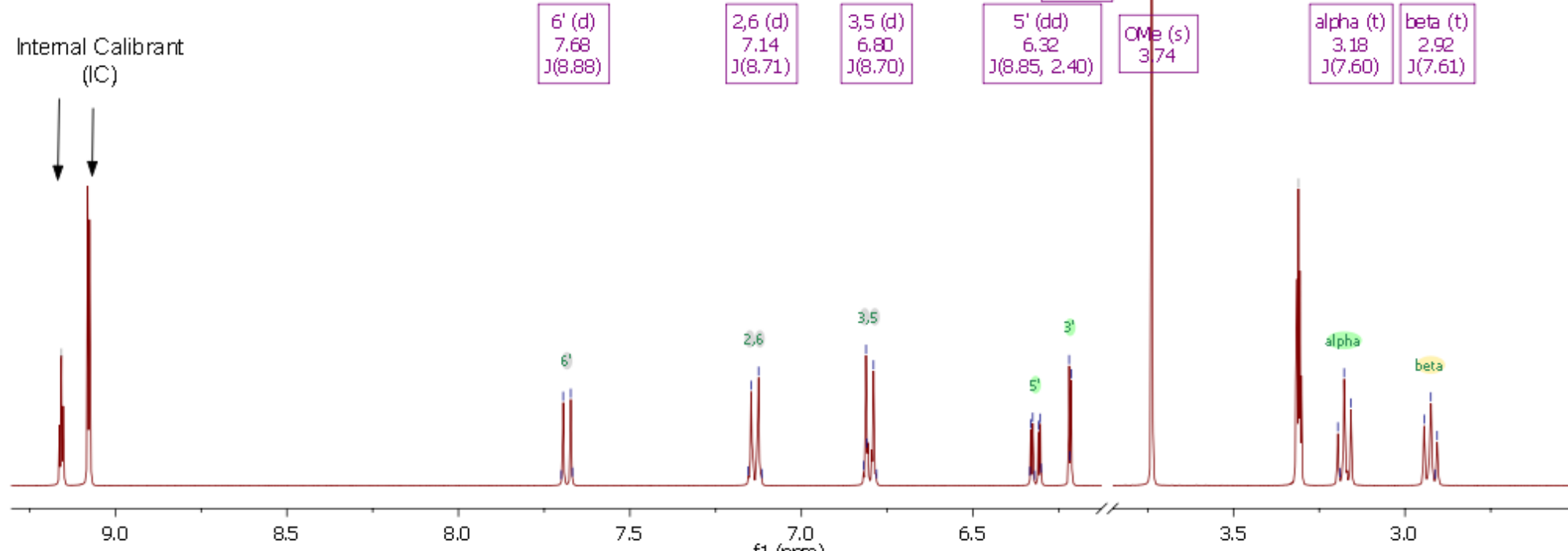

Also freely available at https://doi.org/10.7910/DVN/QZH2DP (Harvard Dataverse) together with 1D/ ${ }^{13} \mathrm{C}$ and 2D NMR data and 4-Omethyldavidigenin ${ }^{1} \mathrm{H}$ full spin analysis enabling the accurate description of ${ }^{1} \mathrm{H}$ resonances, and coupling constants. 
2. Purity determination of synthetic 4-O-methyldavidigenin (compound 2 ):

The purity of the biologically tested synthetic 4-O-methyldavidigenin was determined by quantitative ${ }^{1} \mathrm{H}$ NMR analysis with internal calibrant (IC), as follows: synthetic 4-O-methyldavidigenin $(2.02 \mathrm{mg})$ was precisely weighed with a Mettler Toledo XS105 Dual Range analytical balance and dissolved in $200 \mu \mathrm{L}$ of $\mathrm{CD}_{3} \mathrm{OD}$ containing $8.98 \mathrm{mg} / \mathrm{mL}$ of 3,5-dinitrobenzoic acid (Fluka, TraceCERT, purity $99.54 \% \mathrm{w} / \mathrm{w}$ lot \# BCBH8381V) as IC. The $1 \mathrm{D}{ }^{1} \mathrm{H}$ NMR spectra were acquired at $25{ }^{\circ} \mathrm{C}$ under quantitative conditions (qHNMR) using a $90^{\circ}$ excitation pulse experiment on a JOEL ECZ $400 \mathrm{MHz}$ instrument equipped with $5 \mathrm{~mm}$ multinuclear Royal probe. A total of 64 scans were collected with a pulse width of $7.17 \mu \mathrm{s}$, a spectral width of $20 \mathrm{ppm}$, and a relaxation delay of $60 \mathrm{~s}$. The purity of synthetic DMC-2 was determined to be $98.95 \% \mathrm{w} / \mathrm{w}$ using the previously described absolute method (1).

(1) Pauli GF, Chen SN, Simmler C, Lankin DC, Godecke T, Jaki BU, Friesen JB, McAlpine JB, Napolitano JG: Importance of purity evaluation and the potential of quantitative (1)H NMR as a purity assay. J Med Chem 2014;57:9220-9231. 
3. MS/MS spectra of 4-O-methyldavidigenin and 4'-O-methyldavidigenin in the KOF (ESI+, 10-20 eV)
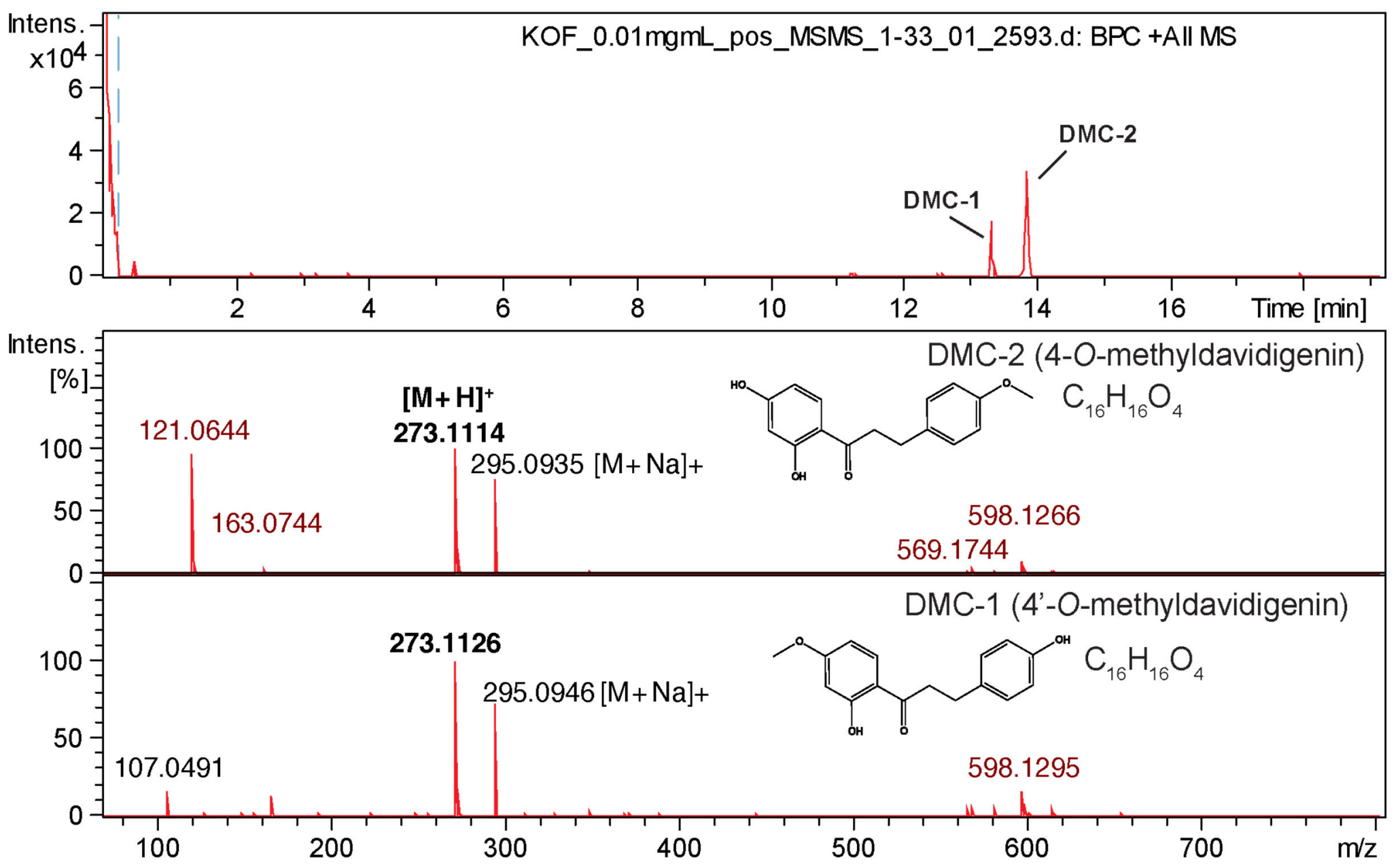

(1) DMC-1: 4'-O-methyldavidigenin (Pubchem CID: 2762958, CHEMBL490352) $[\mathrm{M}+\mathrm{H}]^{+} \mathrm{m} / \mathrm{z}$ calculated for $273.1171, \mathrm{C}_{16} \mathrm{H}_{16} \mathrm{O}_{4}(-1.8 \mathrm{ppm}$ )

(2) DMC-2: 4-O-methyldavidigenin (Pubchem CID: 578444), [M+H] $\left.]^{+}\right]^{+} \mathrm{m} / \mathrm{z}$ calculated for $273.1171, \mathrm{C}_{16} \mathrm{H}_{16} \mathrm{O}_{4}(2.5 \mathrm{ppm})$ 


\section{S10. Comparative ${ }^{1} \mathrm{H}$ NMR spectra of KOF with synthetic 4-O-methyldavidigenin}

(2) DMC-2: 4-O-methyldavidigenin

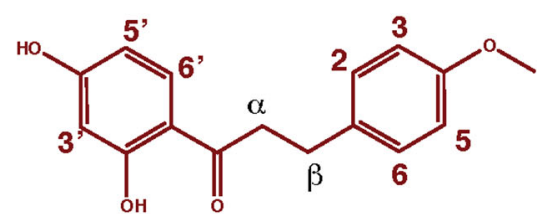

$69.59 \% \mathrm{w} / \mathrm{w}$ of fraction

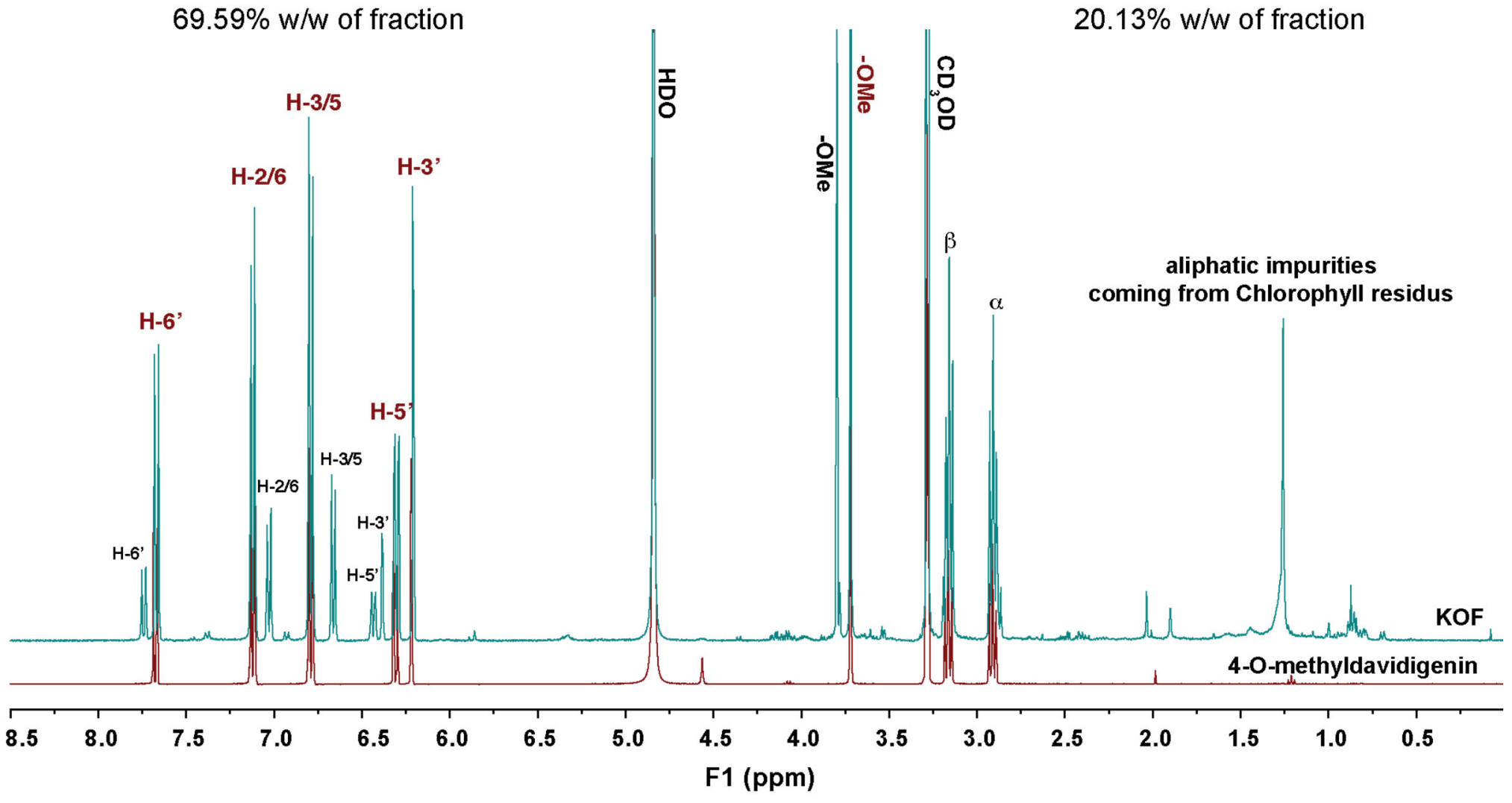

Data acquired under quantitative conditions at $400 \mathrm{MHz}$ in $\mathrm{CD}_{3} \mathrm{OD}$. Also freely available at https://doi.org/10.7910/DVN/QZH2DP (Harvard Dataverse). Quantitative results were obtained using the ECIC method according to Pauli GF et al. J. Med. Chem., 2014, 57 (22) https://doi.org/10.1021/jm500734a . 


\section{S11. Annotated ${ }^{1} \mathrm{H}$ NMR of elemicin and MS/MS spectra of both (iso)-elemicin}

1. Assigned ${ }^{1} \mathrm{H}$ NMR spectrum of elemicin $\left(400 \mathrm{MHz}, \mathrm{CD}_{3} \mathrm{OD}\right)$

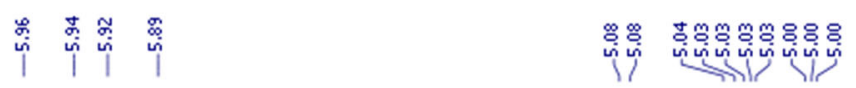

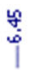

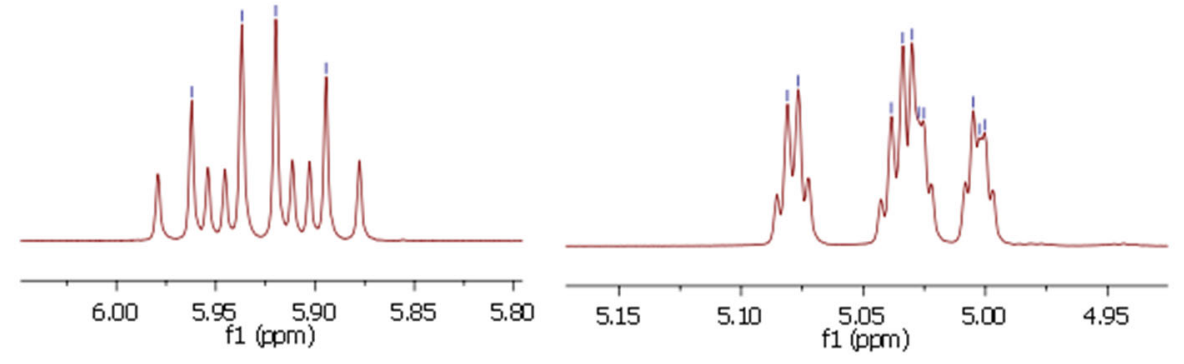

Elemicin ${ }^{1} \mathrm{H} N M R$ spectrum ( $\left.400 \mathrm{MHz}, \mathrm{CD}_{3} \mathrm{OD}\right)$

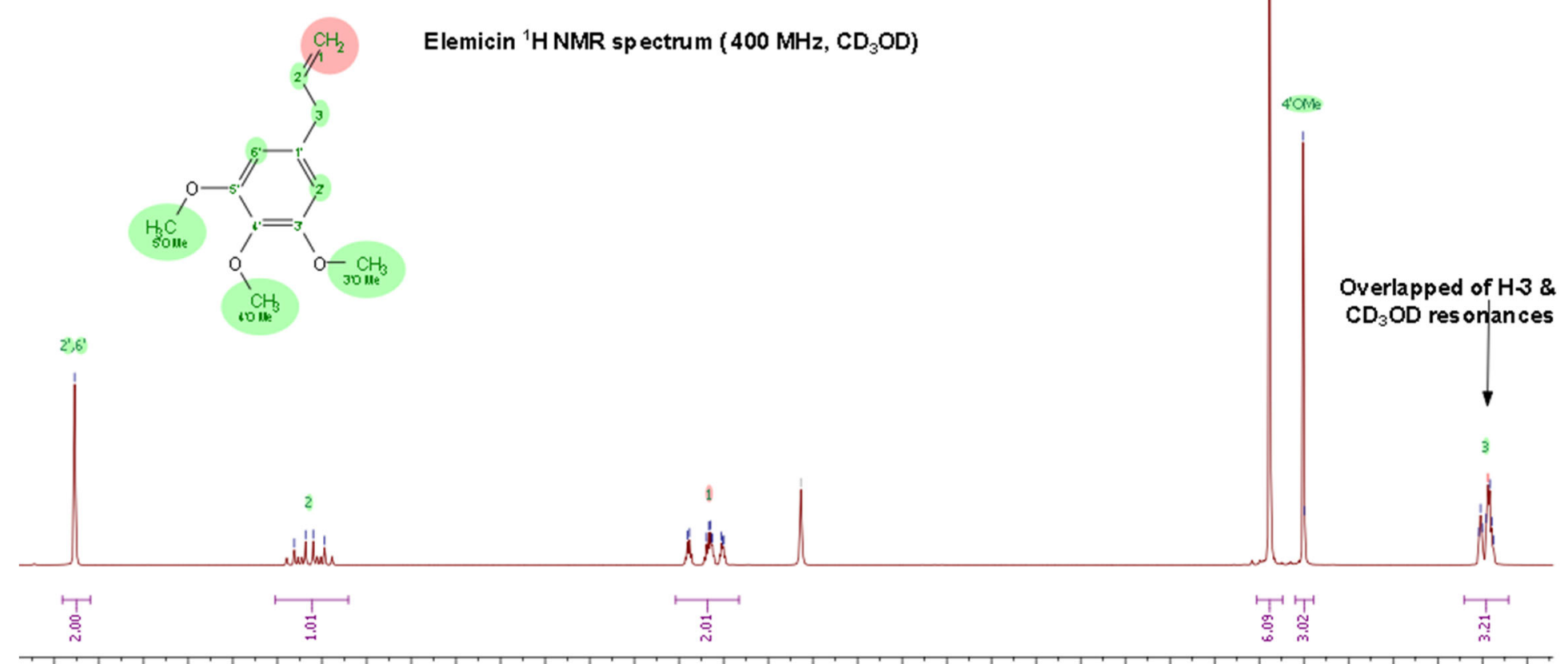

$\begin{array}{lllllllllllllllllllllllllllllllllllll}6.5 & 6.4 & 6.3 & 6.2 & 6.1 & 6.0 & 5.9 & 5.8 & 5.7 & 5.6 & 5.5 & 5.4 & 5.3 & 5.2 & 5.1 & 5.0 & 4.9 & 4.8 & 4.7 & 4.6 & 4.5 & 4.4 & 4.3 & 4.2 & 4.1 & 4.0 & 3.9 & 3.8 & 3.7 & 3.6 & 3.5 & 3.4 & 3.3 & 3.2\end{array}$

Also freely available at https://doi.org/10.7910/DVN/QZH2DP (Harvard Dataverse) together with $1 \mathrm{D} /{ }^{13} \mathrm{C}$ and $2 \mathrm{D}$ NMR data and Elemicin ${ }^{1} \mathrm{H}$ full spin analysis enabling the accurate description of ${ }^{1} \mathrm{H}$ resonances, and coupling constants. 
2. $M S / M S$ spectra of (iso)-elemicin (ESI+, $10-20 \mathrm{eV})$

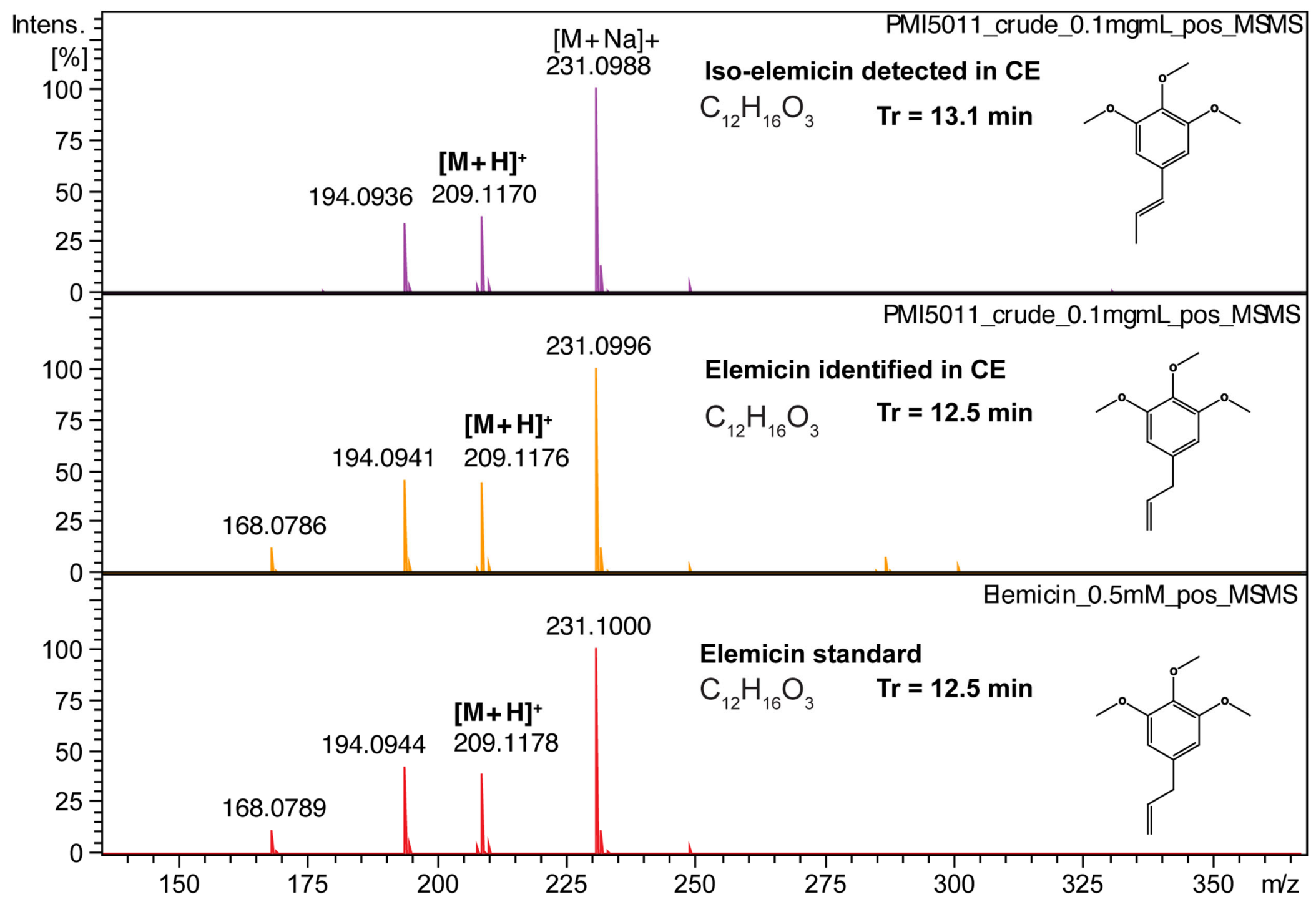

Iso-elemicin ( trans Pubchem CID:636822, cis CID:5281758 ) [M+H] $]^{+} \mathrm{m} / \mathrm{z}$ calculated for 209.1172, $\mathrm{C}_{12} \mathrm{H}_{16} \mathrm{O}_{3}(1.1 \mathrm{ppm})$

Elemicin (Pubchem CID: 10248), [M+H] $]^{+} \mathrm{m} / \mathrm{z}$ calculated for 209.1172, $\mathrm{C}_{12} \mathrm{H}_{16} \mathrm{O}_{3}(-1.8 \mathrm{ppm})$ 
S12. UV spectra of methyldavidigenin isomers, and (Iso)-elemicin
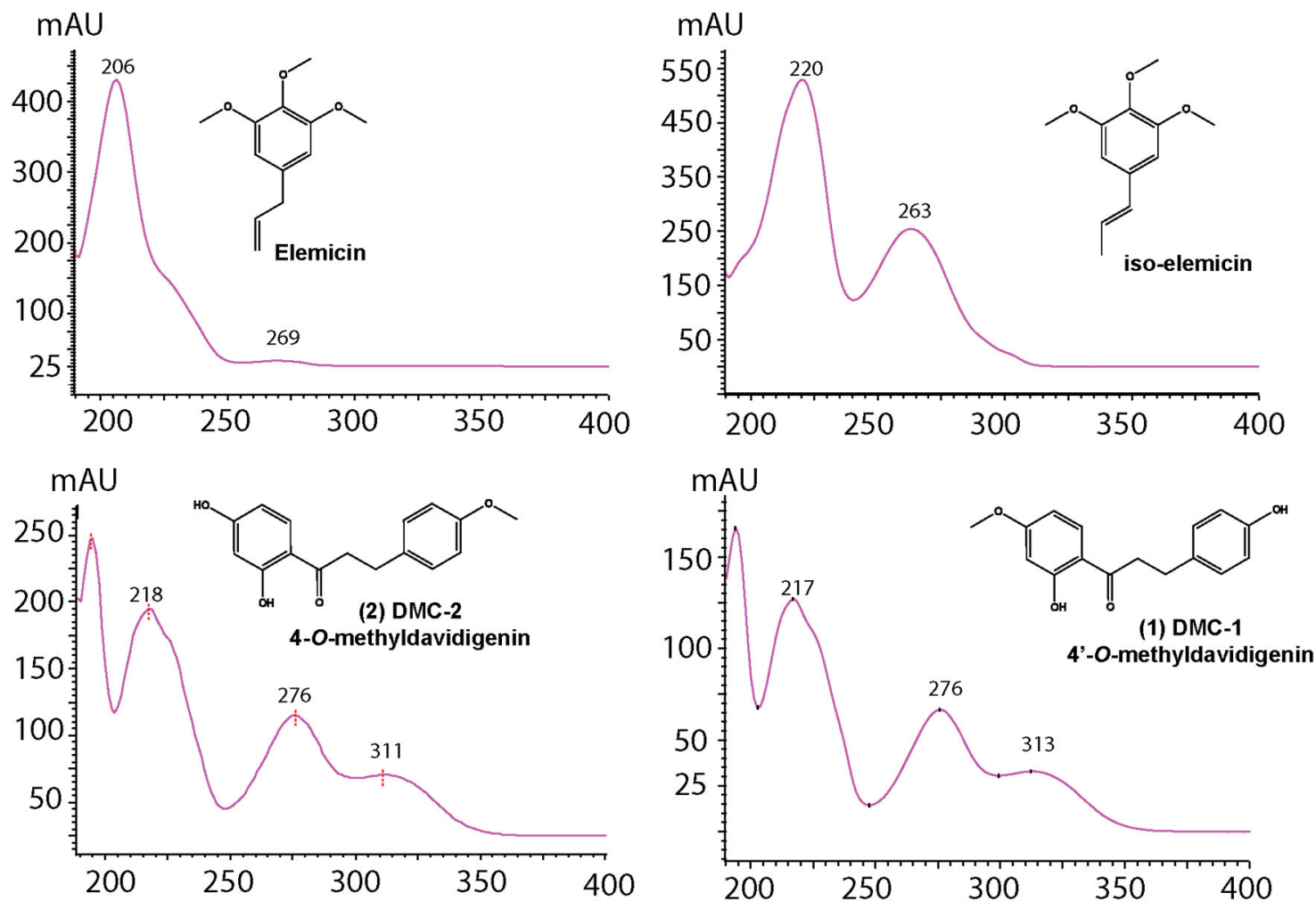

The spectra were obtained from the Shimadzu PDA detector and collected from the UHPLC-UV profile of PMI-5011 crude extract (10 $\mathrm{mg} / \mathrm{mL})$. 
S13. Antibodies used for Western Blot Analyses

\begin{tabular}{|l|c|l|l|l|}
\hline \multicolumn{1}{|c|}{ Primary Antibody } & \multicolumn{1}{c|}{ Type } & Application & \multicolumn{1}{c|}{ Supplier } & \multicolumn{1}{c|}{$\begin{array}{c}\text { Catalog } \\
\text { Number }\end{array}$} \\
\hline Insulin Receptor $\beta$ & Rabbit polyclonal & Western blot & Santa Cruz & sc-711 \\
\hline pTyr $^{1150}$-Insulin Receptor & Mouse monoclonal & Western blot & Millipore & $04-299$ \\
\hline IRS-1 & Rabbit polyclonal & Western blot & Millipore & $05-699$ \\
\hline pTyr $^{608}$-IRS-1 & Rabbit polyclonal & Western blot & Millipore & $09-432$ \\
\hline pSer ${ }^{307}$-IRS-1 & Mouse monoclonal & Western blot & Millipore & $05-1087$ \\
\hline PI-3K p85 & Rabbit monoclonal & Western blot & Abcam & ab133595 \\
\hline AKT $^{308}$ AKT & Mouse monoclonal & Western blot & Cell Signaling & 2920 \\
\hline p-Thr & Rabbit monoclonal & Western blot & Cell Signaling & 4056 \\
\hline p-Ser ${ }^{473}$ AKT & Mouse monoclonal & Western blot & Cell Signaling & 4051 \\
\hline mTOR & Mouse monoclonal & Western blot & Santa Cruz & sc-136269 \\
\hline p-Ser ${ }^{2448}$ mTOR & Rabbit monoclonal & Western blot & Cell Signaling & 2976 \\
\hline AS160 & Rabbit monoclonal & Western blot & Cell Signaling & 2670 \\
\hline p-Thr ${ }^{642}$ As160 & Rabbit polyclonal & Western blot & Millipore & $07-802$ \\
\hline Glut4 & Mouse monoclonal & Western blot & R\&D Systems & MAB1262 \\
\hline FOXO3a & Rabbit monoclonal & Western blot & Cell Signaling & 2497 \\
\hline p-Ser ${ }^{253}$ FOXO3a & Rabbit polyclonal & Western blot & Millipore & $06-953$ \\
\hline GSK3 $\beta$ & Mouse monoclonal & Western blot & Cell Signaling & 9832 \\
\hline p-GSK3 $\alpha / \beta$ & Rabbit polyclonal & Western blot & Cell Signaling & 9331 \\
\hline$\beta$-actin & Western blot & Santa Cruz & sc-47778 \\
\hline
\end{tabular}

